\title{
Trehalose: effect on cryopreservation of umbilical cord blood-derived hematopoietic stem cells
}

\author{
Santwana Mantri $^{1 *}$, Ashok K. Satpathy ${ }^{2}$, Prakash C. Mohapatra ${ }^{3}$ \\ ${ }^{1}$ MRU, SCB Medical College, and Hospital, Cuttack (Odisha), India \\ ${ }^{2}$ Regional Medical Research Center (ICMR), Chandrasekharpur, Odisha, India \\ ${ }^{3}$ Department of Bio-Chemistry, S.C.B. Medical College Hospital, Cuttack, India
}

\begin{abstract}
Introduction: Concerns over dimethyl sulfoxide (DMSO) toxicity, related to adverse reactions after hematopoietic stem cells (HSCs) therapy, warrant the development of an optimized DMSO-free or DMSO-reduced cryopreservation protocol for the quality and safety of HSCs.

In this regard, the ideal concentration of trehalose, as a non-toxic natural cryoprotectant, is still an area of research. Based on the outcome of our previous study on lower concentrations of trehalose, the present study was focused on evaluating its cryoprotective efficacy at an increased concentration (0.5 M) on HSCs compared to $10 \%$ DMSO. This is a laboratory-based experimental study.

Material and methods: The separated mononuclear cells collected from umbilical cord blood were set for culture up to two passages to get HSCs. The two different concentrations of trehalose, with and without 5\% DMSO, were considered as freezing media for the preservation of the harvested HSCs in a slow freezing set up. Two sequential functional assays, viability followed by hematopoietic colony-forming unit assay, were performed with post-thawed cells of freezing media used in this study. Seventeen cord blood samples were selected.
\end{abstract}

Results: Study results revealed $0.5 \mathrm{M}$ trehalose and DMSO $5 \%$ showed the highest viability of $91.8 \pm 2.8 \%$ of HSCs. $5 \%$ DMSO inclusion to trehalose $(0.5 \mathrm{M})$ ameliorated hematopoietic colonies such as erythroid and myeloid colonies with no significant difference from that of $10 \%$ DMSO.

Conclusion: $0.5 \mathrm{M}$ trehalose has proved to be a better concentration than 10\% DMSO alone. This experimental study needs further transplantation-based clinical trials using post-thawed cells to ensure the safety of preserved HSCs from cord blood and other sources.

Key words: DMSO, cord blood, trehalose, cryoprotective media

Acta Haematologica Polonica 2021; 52, 2: 121-126

\section{Introduction}

The clinical utility of cryopreserved hematopoietic stem cells (HSCs) faces challenges related to the quality and safety of cellular products. The effective therapeutic use of cryopreserved HSCs depends on the ideal cryoprotective behavior of cryoprotective agents (CPAs) or cryoprotectants [1]. Moreover, several adverse reactions after transplantation have been associated with standard dimethyl sulfoxide (DMSO) $(10 \%)$ inclusion as CPA for cryopreservation of stem cells $[2,3]$. As DMSO toxicity to cells is concentration-dependent, even its absence in the freezing medium, or total removal

\footnotetext{
*Address for correspondence: Santwana Mantri,

MRU, SCB Medical College, and Hospital,

Cuttack (Odisha), India-753007, phone: +91 993455646 ,

e-mail: sshmantri@gmail.com

Received: 31.03.2020

Accepted: 26.10.2020
} 
before transfusion, would not be better in terms of the need to reduce toxicity $[4,5]$. The preclinical evaluation of an automated sequential washing procedure for pot-thaw DMSO exclusion in HPC grafts has proven to be effective [6].

DMSO removal from long-term frozen PBSC grafts using a fully automated, closed system washing device has been shown to result in a high recovery of viable $\mathrm{CD} 34^{+}$cells [7]. Additionally, reduced concentration of DMSO (5\%) alone has been recommended to enhance the kinetics of engraftment [8], which can be further lessened by adopting a combination of DMSO with another cryoprotectant solution [9]. Complex carbohydrates such as hydroxy ethyl starch (HES) and dextran, the synthetic polymer of glucose, are used as cryoprotectants along with DMSO at their different concentrations for various cell types. Many studies have adopted a standardized combination of $5 \%$ DMSO with $6 \%$ HES and $10 \%$ DMSO with a $2 \%$ dextran freezing medium. But improved post-thaw viability and hematopoietic functionality of CB stem cells have been demonstrated by a freezing medium containing 5\% DMSO and 5\% pentastarch HES [10], rather than 10\% DMSO [11].

Furthermore, the safety of using HES alone as a cryoprotectant or in combination with other CPAs has been reviewed [12], though HES is less toxic than DMSO. Even these synthetic colloids are known to be associated with adverse drug reactions when used for several therapeutic purposes $[13,14]$, warranting the safety of cellular products used for transfusion.

In this regard, natural, non-toxic sugars such as trehalose and sucrose come under the spotlight because they are used by organisms to combat overstress conditions such as heat, cold, and desiccation sugars [15]. These disaccharides are used as CPA with and without DMSO [16]. In our earlier study, the cryoprotective effect of these two sugars at only low concentrations was very poor, and improved with $2 \%$ DMSO inclusion for cord blood-derived mononuclear cells (MNCs) [17]. But $10 \%$ of DMSO was not taken as a variable.

Hence the above findings pave the way for the assessment of the cryoprotective behavior of trehalose at its higher concentration $(0.5 \mathrm{M})$ with or without the inclusion of $5 \%$ DMSO rather than $10 \%$ DMSO, using a conventional slow cooling method for cultured HSCs, because chemical toxicity and extreme osmotic stress conditions are associated with vitrification. Colony forming unit (CFU) has been recommended as being an excellent measure of viable stem cells and cell division and colony formation over time [18], something which aligns with the preclinical quality assessment of post-thawed products in our study.

\section{Materials and methods}

\section{Experimental design}

This present study was designed to assess the cryoprotective effectiveness of trehalose on harvested HSCs (derived from UCB), compared to standard 10\% DMSO.
Our study objective was accomplished in four sequential phases:

- in vitro culture of MNCs (isolated from $\mathrm{CB}$ ) for the yield of HSCs;

- cryopreservation of HSCs with six different freezing media i.e. media containing trehalose $(0.2 \mathrm{M}, 0.5 \mathrm{M})$ alone, $5 \%$ DMSO in combination with trehalose $(0.2 \mathrm{M}$, $0.5 \mathrm{M}), 5 \%$ DMSO, and conventional 10\% DMSO alone;

- post thawed viability analysis of cryopreserved HSCs of the six participating freezing media by taking five different measurements (replicates) for each one;

- hematopoietic functionality assay of the post thawed HSCs of freezing media (showing superior post-thawed HSCs viability) by considering three different measurements for each one.

The current study is an unpublished part of project work funded by the Department of Science \& Technology (DST), Government of India. It was approved by Institutional Ethical Committee (IEC), S.C.B. Medical College, Cuttack, approval no. IEC.157/30.08.2012. CB collection was performed (irrespective of the gender of the newborn) from the Department of Obstetrics and Gynecology with informed consent from mothers. While it was processing, an in vitro culture of HSCs and cryopreservation studies were accomplished in the Department of Biochemistry, S.C.B. Medical College, Cuttack. Samples of cords of full-term deliveries from cesarean sections were preferred as sample collection from vaginal deliveries in the labour room was mostly associated with microbial contamination due to imperfect hygiene.

\section{Inclusion criteria}

The selection of full-term CB samples was based on the absence of indications such as septicemia, other hematological infections or complications, and viral infections (VDRL, human immunodeficiency, hepatitis B, hepatitis C). Other criteria such as samples having any congenital or chromosomal anomalies, inaccurate/unknown gestational age, and signs of hemolysis, were also excluded.

\section{Isolation of MNCs from CB}

Cord blood (65-70 mL) was collected using citrate-phosphate-dextrose (CPD, Sigma-Aldrich, USA) as an anticoagulant during collection and diluted with phosphate buffered saline A (PBS without $\mathrm{Ca}^{2+} / \mathrm{Mg}^{2+}$, HIMEDIA, India) in a 1:1 ratio. MNCs from $C B$ were recovered from the interface obtained by density gradient centrifugation $(1.077 \mathrm{~g} / \mathrm{mL}$ Histopaque, Sigma). The collected MNCs were washed twice with PBS to remove any residual RBCs. The CB processing time was within seven hours of collection without hemolysis or coagulation. In the present study, we avoided the RBC lysis method which may have some impact on the cell microenvironment during the separation process, affecting cell viability [19]. 


\section{Non-adherent HSCs harvest}

For the primary culture of MNCs, we used a culture medium containing Dulbecco's Modified Eagle's Medium (DMEM, PAN-BIOTECH, Germany), $10 \%$ fetal bovine serum (FBS, PANBIOTECH, Germany), and $1 \%$ penicillin, streptomycin antibiotics (Himedia, India). Cell cultures were carried out at $37^{\circ} \mathrm{C}$ in a humidified atmosphere of $95 \%$ air and $5 \%$ $\mathrm{CO}_{2}$ after plating the cells at a density of $1 \times 10^{6} / \mathrm{cm}^{2}$ in the culture medium. The medium was replaced by a fresh one after $48 \mathrm{~h}$. The floating non-adherent cells of culture were removed from the adherent layer and cultured separately. Culture media having Iscove's Modified Dulbecco's Medium with L-glutamine (IMDM, PAN-BIOTECH, Germany) containing $10 \%$ FBS, $1 \%$ penicillin, streptomycin antibiotics was used for non-adherent cells culture at a seeding density of $1 \times 10^{4} / \mathrm{cm}^{2}$. The culture was fed on alternate days. Media with cultured cells were centrifuged at 1,500 rpm for 5 min after the $7^{\text {th }}$ day of culture. The cells were then washed with PBS-A and counted in a hemocytometer.

\section{Preparation of freezing media}

Based on our current study design, two different concentrations of trehalose, i.e. $0.2 \mathrm{M}$ and $0.5 \mathrm{M}$, were considered for the preparation of four different types of cryoprotective media (as presented in Table I) for evaluation of their optimal cryoprotective function. These media were mostly composed of $10 \%$ bovine serum albumin (BSA, PANBIOTECH, Germany) in Dulbecco's Modified Eagle's Medium (DMEM, PAN-BIOTECH, Germany) to which trehalose (Himedia, India) was added alone or with $5 \%$ DMSO. All freezing media were freshly prepared and cooled at $4{ }^{\circ} \mathrm{C}$. The control media were $5 \%$ and $10 \%$ DMSO only.

\section{Freezing and thawing of HSCs}

After the preparation of the abovementioned freezing media, $2 \times 10^{6} \mathrm{HSC}$ were suspended with $1.8 \mathrm{~mL}$ of the freezing medium of each group separately in a $2 \mathrm{~mL}$ of cryovial tube and immediately transferred to an isopropanol container (Tarson) for freezing at $-80^{\circ} \mathrm{C}$ overnight with a cooling rate of $1^{\circ} \mathrm{C} / \mathrm{min}$. Finally, the isopropanol container was transferred to the canister and stored in the vapor phase of liquid nitrogen $\left(\right.$ at $-156^{\circ} \mathrm{C}$ ) for one month until further analysis.

To thaw the cryopreserved samples, DMEM media with heat-inactivated $10 \%$ fetal bovine serum (FBS) was used. The cryovials, removed from the liquid nitrogen tank, were immediately placed in a water bath maintained at $37^{\circ} \mathrm{C}$ until ice crystals started to disappear and transferred to a $50 \mathrm{ml}$ centrifuge tube, with the thawing medium added slowly in the ratio of 2:1 to the cell suspension. The HSCs were recovered by centrifugation at 1,500 rpm for $6 \mathrm{~min}$ at room temperature. Excess supernatant was decanted and the cell pellet was suspended in the above prewarmed (warmed for $5-6 \mathrm{~min}$ at a temperature of $37^{\circ} \mathrm{C}$ ) thawing
Table I. Preparation of two different groups of disaccharide-based freezing media in combination, with and without $5 \%$ dimethyl sulfoxide (DMSO) for cryopreservation

\begin{tabular}{|l|l|}
\hline Group I freezing media & Group Il freezing media \\
\hline Disaccharides alone & $\begin{array}{l}\text { Disaccharide media } \\
\text { with introduction of 5\% DMSO }\end{array}$ \\
\hline $0.2 \mathrm{M}$ trehalose & $0.2 \mathrm{M}$ trehalose with 5\% DMSO \\
$0.5 \mathrm{M}$ trehalose & $0.5 \mathrm{M}$ trehalose with 5\% DMSO \\
\hline
\end{tabular}

medium. Further washing of recovered HSCs was done twice with thawing media by centrifugation. Cryopreservation and post-thaw viability assay of preserved HSCs were performed in five replicates.

Cell viability was calculated using the following formula:

$\%$ viability $=$ the number of viable post-thawed HSCs $\times 100 /$ total number of HSCs before freezing

\section{Colony forming unit (CFU) assay \\ Hematopoietic colony-forming unit assay}

For the assessment of the functional activity of post thawed HSCs, a colony-forming unit assay was performed. Cell dose of approximately $2 \times 10^{5}$ cells was set for culture by incubating the cells in methylcellulose medium, provided in the kit (Stem Cell Technologies, Vancouver, BC, Canada) containing hematopoietic growth factors, for two weeks according to the standard procedures [20]. Scoring of generated hematopoietic colonies was based on their relative distribution in each culture plate after two weeks (as stated in the information sheet of the kit). Hematopoietic colonies such as erythroid lineage-based burst forming unit-erythroid/colony forming unit-erythroid (BFU-E/CFU-E), myeloid lineage-based colony forming unit-granulocyte, macrophage (CFU-GM), and the mixed type colony-forming unit-granulocyte, erythroid, macrophage, megakaryocyte (CFU-GEMM), were scored under an inverted microscope.

\section{Statistical analysis}

Statistical data was presented as mean \pm standard deviation (SD) and analyzed by ANOVA and Student's t-test with significant $p$ value $<0.05$, using GraphPad PRISM 5 software.

\section{Results}

Twenty cord blood samples were selected according to the study inclusion criteria. During transportation from the Department of Obstetrics and Gynecology to the laboratory for sample processing, hemolysis occurred in three samples. The mean volume of total studied samples was 65.31 $\pm 4.55 \mathrm{~mL}$, and the mean number of MNCs obtained after density gradient centrifugation was $0.602 \pm 0.06 \times 10^{9}$.

Following cryopreservation, optimum scoring of the freezing media was based on post-thaw cell viability assay. 
A clonogenic CFU assay for each optimized medium was performed in triplicates only (a total of $12 \mathrm{CFU}$ assays).

\section{Post-thaw viability of HSCs}

The highest viability percentage, of $91.8 \pm 2.86 \%$, was demonstrated by the freezing medium containing $0.5 \mathrm{M}$ trehalose and DMSO (5\%) with no significant difference from that of $10 \%$ DMSO ( $p=0.4433$ ), as set out in Figure 1 . Trehalose alone revealed better post-thaw cell viability at its higher concentration $(0.5 \mathrm{M})$ than the lower one $(0.2 \mathrm{M})$ with $p<0.05$. A similar outcome was observed even after the addition of DMSO to both of its concentrations. Moreover, post-thaw viability was significantly higher in the case of $0.5 \mathrm{M}$ trehalose with $5 \%$ DMSO medium compared to $5 \%$ DMSO alone $(p<0.0001)$.

\section{Hematopoietic functional outcome}

Optimal post-thaw viability displayed by the trehalose $(0.5 \mathrm{M})$ based freezing medium with and without $5 \%$ DMSO weas selected for CFU Assay. After 14 days of incubation with methylcellulose medium, three types of colonies, BFU-E/ /CFU-E, CFU-GM, and CFU-GEMM, were developed in each culture plate. The total number of hematopoietic colonies (i.e. adding up the BFU/CFU, CFU-GM and CFU-GEMM colonies) produced by post-thawed HSCs of each freezing medium, are set out as mean (SD) in Table II. Here, for each medium, three different measurements were considered for the total number of hematopoietic colonies.

The presence of DMSO in $0.5 \mathrm{M}$ trehalose media ameliorated the total number of hematopoietic colonies (mean \pm SD) rather than its complete absence (Table II). A similar effect was pronounced during individual hematopoietic colony scoring (Figure 2). $0.5 \mathrm{M}$ trehalose exhibited more myeloid lineage-based (CFU-GM) and less mixed (CFU-GEMM) colonies than 5\% DMSO, with $p=0.008$ and $p=0.001$ respectively. But there was a significant difference in the yield of both myeloid $(p<0.0001)$ and erythroid $(p=0.0002)$ colonies in trehalose media only after the inclusion of $5 \%$ DMSO compared to 5\% DMSO alone.

The functional activity of post-thawed HSCs preserved in a conventional freezing medium of $10 \%$ DMSO was compared to that of $0.5 \mathrm{M}$ trehalose in combination with $5 \%$ DMSO. No significant differences were found in the number of erythroid $(p=0.0832)$, myeloid $(p=0.1121)$, or mixed type $(p=0.3611)$ colonies.

\section{Discussion}

To address the contradictory consequence of both cryoprotection and toxicity resulting from routine use of a standard DMSO dose (10\%), additional CPAs like natural disaccharides remain the focus of discussion. The dipole potential of the cell membrane is decreased with an increasing concentration of trehalose owing to its bioprotective function

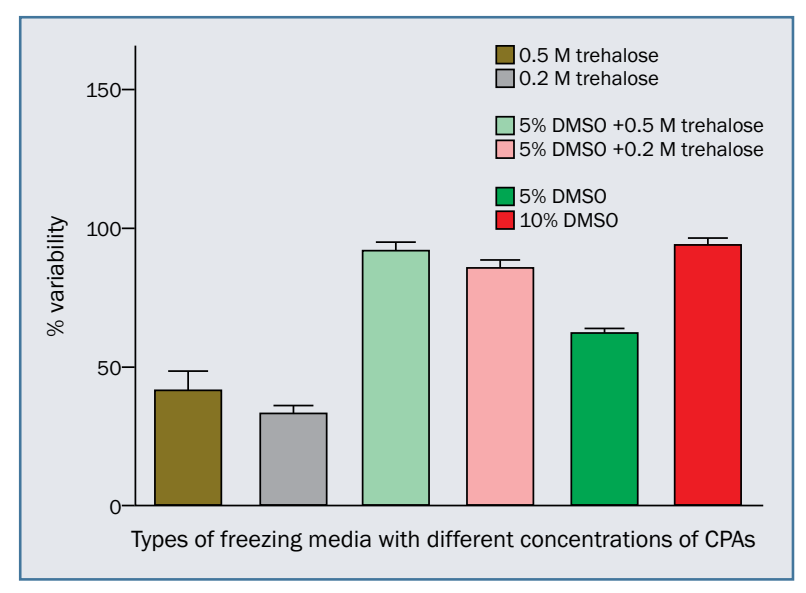

Figure 1. Viability percentages as shown by different media. All data represented as mean \pm standard deviation for five replicates; CPAs - cryoprotective agents; DMSO - dimethyl sulfoxide

Table II. Total number of hematopoietic colonies as generated by different cryoprotective media used

\begin{tabular}{|l|c|}
\hline Type of cryoprotective medium & $\begin{array}{c}\text { Total number of colonies } \\
(\text { mean } \pm \text { SD) }\end{array}$ \\
\hline 10\% DMSO & $256 \pm 5.51$ \\
\hline $5 \%$ DMSO & $138 \pm 4.51$ \\
0.5 M trehalose & $154 \pm 4.73$ \\
0.5 M trehalose $+5 \%$ DMSO & $276 \pm 9.07$ \\
\hline All data represented as mean \pm standard deviation (SD) for triplicates; DMSO - dimethyl
\end{tabular}
sulfoxide

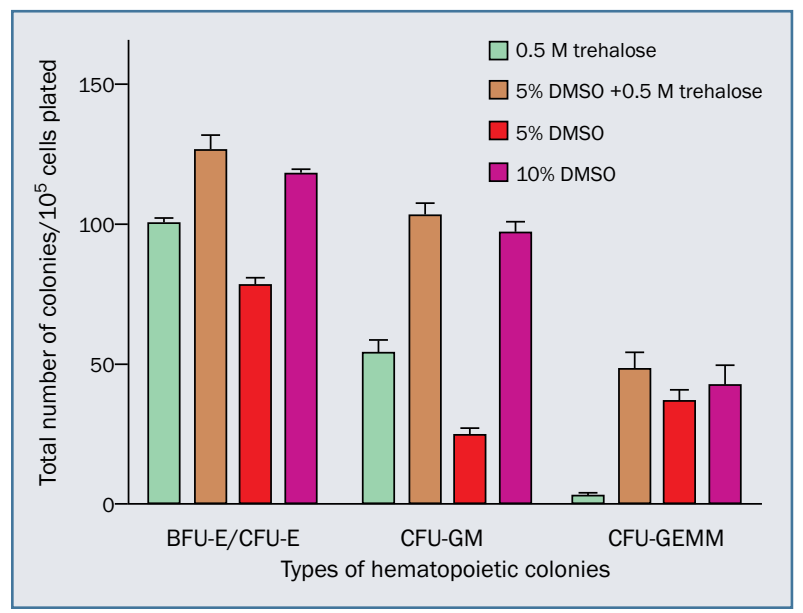

Figure 2. Comparison of three types of hematopoietic colonies distribution during colony forming unit (CFU) assay. All data represented as mean \pm standard deviation (SD) of three different measurements for each freezing medium; BFU-E/CFU-E - burst forming unit-erythroid/colony forming unit-erythroid; DMSO - dimethyl sulfoxide; CFU-GM - myeloid lineage-based colony forming unit-granulocyte, macrophage; CFU-GEMM colony-forming unit-granulocyte, erythroid, macrophage, megakaryocyte 
[21], which was observed by increasing the concentration of trehalose from $0.2 \mathrm{M}$ to $0.5 \mathrm{M}$ in our study. Furthermore, the conventional method of DMSO removal comprises the repeated process of immediate centrifugation of thawed cell products, the removal of the supernatant, and the addition of fresh solution to the cell population. We adopted this traditional procedure for washing thawed products instead of new technology-based washing devices, which already exhibit high recovery of viable $\mathrm{CD} 34^{+}$cells, with good engraftment potential in some of the studies [22, 23]. Volume reduction in DMSO concentration still does not have any impact on engraftment after autologous peripheral blood stem cell (PBSC) transplantation as reported by one of the clinical studies [24].

The impact of trehalose at its $0.5 \mathrm{M}$ concentration alone contributes similar results to those of $10 \%$ DMSO in the preservation of stem cells derived from BM and PB [25]. The reported results are not in agreement with the current data. Conversely, post-thawed assessment outcomes of existing data exhibit efficient cryoprotection only by combined media of trehalose and DMSO on cord blood HSCs. One of the recent relevant studies on PBSCs demonstrates improved cryoprotection by $1 \mathrm{M}$ trehalose alone to $\mathrm{CD} 34^{+}$cells compared to the standard freezing procedure that uses 10\% DMSO [16], where the cut off value of trehalose concentration is $1 \mathrm{M}$ without the use of DMSO and catalase.

The quality of post-thawed HSCs involves phenotypical characterization of cells by flow-cytometric analysis to determine cell viability (viability dye 7-AAD) and cell type (CD 34 count) and furthermore by CFU analysis.

But post-thaw viability of cells does not guarantee post-thaw cell functionality.

Delayed engraftments have been observed regardless of adequate post-thaw viability of $\mathrm{CD} 34^{+}$cells [26]. Post-thaw cell functionality can be assessed by the CFU test, as this is a decisive step before heading for clinical trials [27]. This is a powerful measure of engraftment related to UCB transplantation [18].

The results of the functional assay (CFU) of post thawed cells in the current study confirm that $0.5 \mathrm{M}$ trehalose in association with $5 \%$ DMSO can be an ideal cryoprotective solution, and could act as a valuable alternative to the typical 10\% DMSO medium.

This simple cryopreservation method needs further clinical trials based on investigations to ascertain the safety of using preserved HSCs for myriad diseases in a tertiary care center setting, because it is a cost-effective cryopreservation protocol.

\section{Limitations}

Time programmed freezing methods and new technology-based well-established washing devices were not used for this study due to inadequate allocated funds.

\section{Acknowledgments}

This study was an unpublished part of project work funded by the Department of Science \& Technology (DST), Government of India.

\section{Conflict of interest}

None.

\section{Financial support \\ None.}

\section{Ethics}

The work described in this article has been carried out in accordance with the Code of Ethics of the World Medical Association (Declaration of Helsinki) for experiments involving humans; EU Directive 2010/63/EU for animal experiments; Uniform Requirements for Manuscripts submitted to Biomedical Journals

\section{References}

1. Hornberger K, Yu G, McKenna D, et al. Cryopreservation of hematopoietic stem cells: emerging assays, cryoprotectant agents, and technology to improve outcomes. Transfus Med Hemother. 2019; 46(3): 188-196, doi: 10.1159/000496068, indexed in Pubmed: 31244587.

2. Ruiz-Delgado GJ, Mancías-Guerra C, Tamez-Gómez EL, et al. Dimethyl sulfoxide-induced toxicity in cord blood stem cell transplantation: report of three cases and review of the literature. Acta Haematol. 2009; 122(1): 1-5, doi: 10.1159/000227267, indexed in Pubmed: 19590176.

3. Shu Z, Heimfeld S, Gao D. Hematopoietic SCT with cryopreserved grafts: adverse reactions after transplantation and cryoprotectant removal before infusion. Bone Marrow Transplantation. 2013; 49(4): 469-476, doi: 10.1038/bmt.2013.152.

4. Hirata Y, Kishino K, Onozaki F, et al. Use of cryoprotectant-depleted allogeneic peripheral blood stem cells for transplantation. Hematology. 2011; 16(4): 221-224, doi: 10.1179/102453311X1302556894166 4, indexed in Pubmed: 21756538.

5. Scerpa MC, Daniele N, Landi F, et al. Automated washing of human progenitor cells: evaluation of apoptosis and cell necrosis. Transfus Med. 2011; 21(6): 402-407, doi: 10.1111/j.1365-3148.2011.01103.x, indexed in Pubmed: 22032614.

6. Abonnenc M, Pesse B, Tissot JD, et al. Automatic washing of thawed haematopoietic progenitor cell grafts: a preclinical evaluation. Vox Sang. 2017; 112(4): 367-378, doi: 10.1111/vox.12503, indexed in Pubmed: 28337763.

7. Aerts-Kaya F, Koca G, Sharafi P, et al. Automated washing of long-term cryopreserved peripheral blood stem cells promotes cell viability and preserves CD34+ cell numbers. Bone Marrow Transplant. 2018; 53(9): 1225-1227, doi: 10.1038/s41409-018-0192-7, indexed in Pubmed: 29703970.

8. Galmes A, Gutiérrez A, Sampol A, et al. Long-term hematological reconstitution and clinical evaluation of autologous peripheral blood stem cell transplantation after cryopreservation of cells with $5 \%$ and $10 \%$ dimethylsulfoxide at -80 degrees $C$ in a mechanical freezer. Haematologica. 2007; 92(7): 986-989, doi: 10.3324/haematol.11060, indexed in Pubmed: 17606452. 
9. Rowley SD, Feng Z, Chen L, et al. A randomized phase III clinical trial of autologous blood stem cell transplantation comparing cryopreservation using dimethylsulfoxide vs dimethylsulfoxide with hydroxyethylstarch. Bone Marrow Transplant. 2003; 31(11): 1043-1051, doi: 10.1038/sj.bmt.1704030, indexed in Pubmed: 12774058.

10. Hayakawa J, Joyal EG, Gildner JF, et al. 5\% dimethyl sulfoxide (DMSO) and pentastarch improves cryopreservation of cord blood cells over 10\% DMSO. Transfusion. 2010; 50(10): 2158-2166, doi: 10.1111/j.1537-2995.2010.02684.x, indexed in Pubmed: 20492608 .

11. Mitrus I, Smagur A, Giebel S, et al. A faster reconstitution of hematopoiesis after autologous transplantation of hematopoietic cells cryopreserved in $7.5 \%$ dimethyl sulfoxide if compared to $10 \%$ dimethyl sulfoxide containing medium. Cryobiology. 2013; 67(3): 327-331, doi: 10.1016/j.cryobiol.2013.09.167, indexed in Pubmed: 24125911.

12. Stolzing A, Naaldijk $Y$, Fedorova $V$, et al. Hydroxyethylstarch in cryopreservation - mechanisms, benefits and problems. Transfusion and Apheresis Science. 2012; 46(2): 137-147, doi: 10.1016/j. transci.2012.01.007.

13. https://www.sciencedirect.com/topics/medicine-and-dentistry/hydroxyethyl-starch (March 26, 2020).

14. https://www.sciencedirect.com/topics/neuroscience/dextran-40 (March 26, 2020).

15. Jain NK, Roy I. Effect of trehalose on protein structure. Protein Sci. 2009; 18(1): 24-36, doi: 10.1002/pro.3, indexed in Pubmed: 19177348.

16. Martinetti D, Colarossi C, Buccheri S, et al. Effect of trehalose on cryopreservation of pure peripheral blood stem cells. Biomed Rep. 2017; 6(3): 314-318, doi: 10.3892/br.2017.859, indexed in Pubmed: 28451392

17. Mantri S, Kanungo S, Mohapatra PC. Cryoprotective effect of disaccharides on cord blood stem cells with minimal use of DMSO. Indian J Hematol Blood Transfus. 2015; 31(2): 206-212, doi: 10.1007/ /s12288-014-0352-x, indexed in Pubmed: 25825559.

18. Page KM, Zhang L, Mendizabal A, et al. Total colony-forming units are a strong, independent predictor of neutrophil and platelet engraftment after unrelated umbilical cord blood transplantation: a single-center analysis of 435 cord blood transplants. Biol Blood Marrow Transplant. 2011; 17(9): 1362-1374, doi: 10.1016/j.bbmt.2011.01.011, indexed in Pubmed: 21277377.
19. Keong CK, Nadarajah VD, Lee TJu. Development of a purification method of pure primary lymphocytes for cell viability assays. Malays J Med Sci. 2007; 14(1): 38-45, indexed in Pubmed: 22593650.

20. Mahmut N, Katayama Y, Takenaka K, et al. Analysis of circulating hematopoietic progenitor cells after peripheral blood stem cell transplantation. Int J Hematol. 1999; 69(1): 36-42, indexed in Pubmed: 10641441.

21. Luzardo MC, Amalfa F, Nuñez AM, et al. Effect of trehalose and sucrose on the hydration and dipole potential of lipid bilayers. Biophys $\mathrm{J}$. 2000; 78(5): 2452-2458, doi: 10.1016/s0006-3495(00)76789-0.

22. Mfarrej B, Bouchet G, Couquiaud J, et al. Pre-clinical assessment of the Lovo device for dimethyl sulfoxide removal and cell concentration in thawed hematopoietic progenitor cell grafts. Cytotherapy. 2017; 19(12): 1501-1508, doi: 10.1016/j.jcyt.2017.09.001, indexed in Pubmed: 29037941.

23. Aerts-Kaya F, Koca G, Sharafi P, et al. Automated washing of long-term cryopreserved peripheral blood stem cells promotes cell viability and preserves CD34+ cell numbers. Bone Marrow Transplant. 2018; 53(9): 1225-1227, doi: 10.1038/s41409-018-0192-7, indexed in Pubmed: 29703970.

24. Mitrus I, Smagur A, Fidyk W, et al. Reduction of DMSO concentration in cryopreservation mixture from $10 \%$ to $7.5 \%$ and $5 \%$ has no impact on engraftment after autologous peripheral blood stem cell transplantation: results of a prospective, randomized study. Bone Marrow Transplant. 2018; 53(3): 274-280, doi: 10.1038/s41409-017-00566, indexed in Pubmed: 29269805.

25. Scheinkönig C, Kappicht S, Kolb HJ, et al. Adoption of long-term cultures to evaluate the cryoprotective potential of trehalose for freezing hematopoietic stem cells. Bone Marrow Transplant. 2004; 34(6): 531-536, doi: 10.1038/sj.bmt.1704631, indexed in Pubmed: 15286692.

26. Morgenstern DA, Ahsan G, Brocklesby M, et al. Post-thaw viability of cryopreserved peripheral blood stem cells (PBSC) does not guarantee functional activity: important implications for quality assurance of stem cell transplant programmes. Br J Haematol. 2016; 174(6): 942-951, doi: 10.1111/bjh.14160, indexed in Pubmed: 27291859.

27. Chevaleyre J, Rodriguez L, Duchez P, et al. A novel procedure to improve functional preservation of hematopoietic stem and progenitor cells in cord blood stored at $+4^{\circ} \mathrm{C}$ before cryopreservation. Stem Cells Dev. 2014; 23(15): 1820-1830, doi: 10.1089/scd.2014.0046, indexed in Pubmed: 24708384. 